\section{Chiasmal apoplexy, an unusual complication of} cerebral glioblastoma

The differential diagnosis of the rapid development of bilateral visual failure includes optic glioma. ${ }^{1,2}$ and meningeal carcinomatosis. ${ }^{3}$ We now describe a case of cerebral glioblastoma complicated by infarction of the optic chiasm.

\section{Case report}

A 70-year-old woman with no past medical history of note initially presented to the physicians with symptoms and signs consistent with a left hemispheric cerebrovascular accident. A right carotid bruit was present, her blood pressure was elevated (200/90 $\mathrm{mmHg}$ ) and she was commenced on metoprolol and aspirin. At this time her visual functions were intact.

Ten weeks later, over a $12 \mathrm{~h}$ period she developed profound bilateral visual loss which was associated with an impairment of her short-term memory. A computed tomographic (CT) head scan was performed, the appearance of which was consistent with a cerebral glioblastoma arising in the left parietal lobe.

On examination the following day, she was normally oriented and had minimal weakness of the right arm and leg. There was no perception of light bilaterally, the pupils were equal and unresponsive, and the ocular movements were full. The remainder of the ocular examination was normal; in particular, the optic discs were neither swollen nor atrophic.

As the visual loss was bilateral, symmetrical and pupil-involving, the lesion was localised to the optic chiasm. The rapidity of the visual deterioration suggested the underlying cause was acute vascular insufficiency. The patient had no symptoms suggestive of giant cell arteritis and the ESR was $41 \mathrm{~mm} / \mathrm{h}$. The magnetic resonance (MR) image is shown in Fig. 1.

A stereotactic biopsy from the left parietal lesion confirmed the tumour to be a glioblastoma multiforme (grade 4 astrocytoma). Two days after the biopsy the patient developed raised intracranial pressure and died. A request for a post-mortem was refused.

\section{Comment}

The visual pathways can be affected by gliomas arising either as a primary event (optic gliomas) or as a result of spread from a cerebral glioma, as in this case.

Optic chiasmal gliomas characteristically present within the first two decades of life with slowly progressive bilateral visual loss. Acute haemorrhage within the tumour can produce precipitous visual loss that can affect one or both eyes and which has been termed 'chiasmal apoplexy'. ${ }^{1}$ Optic gliomas of adulthood present with progressive visual loss, neurological symptoms and death. The pattern of visual loss in those patients in whom the tumour originates intracranially is bilateral, simultaneous and is associated with normalappearing or pale optic discs. ${ }^{2}$

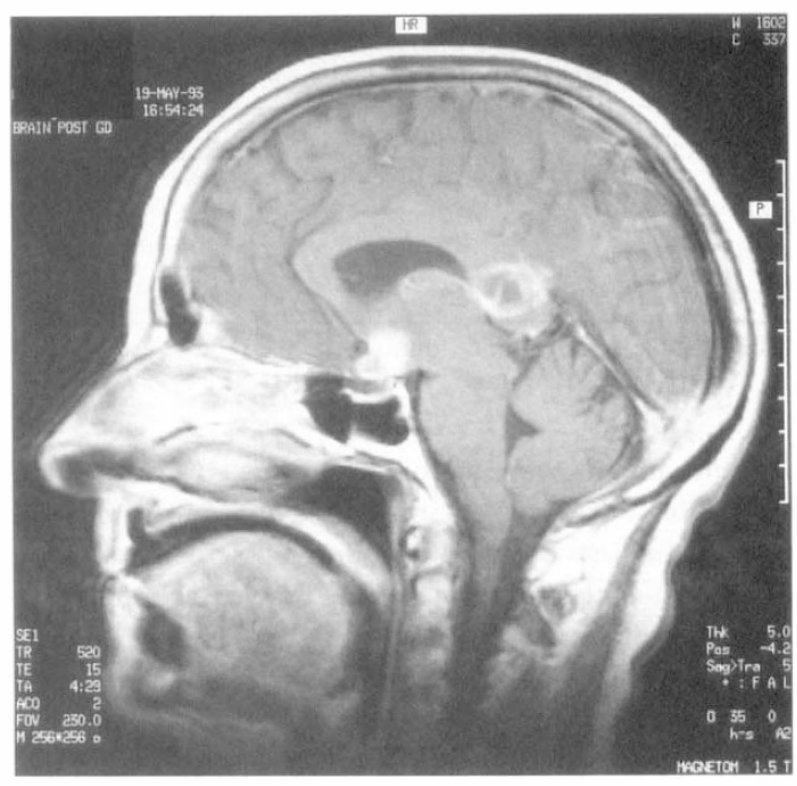

Fig. 1. Sagittal, midline, T1-weighted, post-gadolinium MR scan demonstrating an enhancing cystic mass in the splenium of the corpus callosum. A separate enhancing focus arises from the floor of the third ventricle and assumes the configuration of the chiasmatic and infundibular recesses. Within this focus lies an area of low-density signal that is iso-intense with that of brain and is consistent with infarction of the optic chiasm. The pre-contrast images (not shown) demonstrate the presence of perichiasmal haemorrhage.

Visual loss, which occurs in up to one-third of patients with meningeal carcinomatosis, usually progresses over the course of a few weeks; however, complete loss of vision may occur within $48 \mathrm{~h}^{3}$

Visual symptoms are rarely one of the dominant clinical features of cerebral glioblastoma; even after radiotherapy and chemotherapy less than $10 \%$ of patients survive 2 years. Progressive bilateral visual loss from diffuse infiltration of the anterior visual pathways can be the presenting symptom in patients with gliomatosis cerebri. ${ }^{4}$

Given the extensive nature of the left parietal lobe tumour demonstrated on the CT and MR scans it seems unlikely that the optic chiasm was the primary site of origin of the glioblastoma. Whilst tumour continuity could not be confirmed radiologically, between $5 \%$ and $20 \%$ of all cerebral gliomas are multicentric. The cerebrospinal (CSF) fluid was not examined in our patient; however, meningeal gliomatosis occurs in up to $20 \%$ of supratentorial gliomas. As a rule, these metastases enlarge within the cavity of the ventricle and seldom penetrate deeply into subjacent tissue. It therefore seems unlikely that the perichiasmal glioma demonstrated in Fig. 1 represents evidence of leptomeningeal spread.

In summary, the rapidity of the visual loss experienced by our patient represents acute vascular insufficiency of the optic chiasm. This most likely reflects external compression of the chiasm and its vascular supply by acute haemorrhage occurring within the perichiasmal glioma. 


\section{References}

1. Maitland CG, Abiko S, Hoyt WF, Wilson CB, Okamura T. Chiasmal apoplexy. J Neurosurg 1982;56:118-22.

2. Spoor TC, Kennerdell JS, Martinez AJ, Zorub D. Malignant gliomas of the optic pathways. Am J Ophthalmol 1980;89:284-92.

3. Altrocchi PA, Reinhardt PH, Eckmann PB. Blindness and meningeal carcinomatosis. Arch Ophthalmol 1972;88:508-12.

4. Spector RT, Smith JL, Parker JC Jnr. Cecocentral scotomas in gliomatosis cerebri. J Clin Neuro-ophthalmol 1984;4:229-38.

Mark Wright

Ahmed Kamal

Department of Ophthalmology

Princess Alexandra Eye Pavilion

Chalmers Street

Edinburgh EH3 9HA, UK

lan R. Whittle

Department of Neurosurgery

Western General Hospital

Edinburgh, UK

George T. Vaughan

Department of Neuroradiology

Western General Hospital

Edinburgh, UK

\section{Sir,}

\section{Retinal vein occlusion, the contraceptive pill and the} prothrombin 20210A allele

Retinal vein occlusion is a common ophthalmic disorder the aetiology of which remains unclear. Kirwan and colleagues ${ }^{1}$ claim to have found an association between this and contraceptive pill usage. However, our reanalysis of their data suggests that only 4 of $9(44 \%)$ female patients under 35 years in their series were actually taking the contraceptive pill at the time of the event, compared with $30 \%$ national usage of the pill in this age group - not a significant difference. Furthermore they confined their investigations to markers of arterial disease, rather than venous thrombosis (such as deficiency of antithrombin, protein $C$, protein $S$ or the presence of the factor $\mathrm{V}$ Leiden defect).

Recently a new inherited prothrombotic abnormality has been described that is associated with increased prothrombin levels. ${ }^{2}$ We report a young patient who developed branch retinal vein thrombosis whilst taking the combined oral contraceptive and who was subsequently found to be heterozygous for the prothrombin 20210A allele.

\section{Case report}

A 25-year-old woman presented with a non-ischaemic, macula-sparing branch retinal vein occlusion in her left eye. Her visual acuity was $6 / 6$ and she did not require any specific ophthalmic treatment. At presentation she was taking Dianette, a combined oral contraceptive pill. Four years earlier she had had an episode of drug-related lupus erythematosus induced by tetracycline that resolved completely on withdrawing the medication. Investigation at the time of the retinal vein occlusion showed normal levels of antithrombin, protein C, protein $S$ and anticardiolipin antibodies. The lupus anticoagulant test was negative and the factor $\mathrm{V}$ Leiden was absent. Two years later she was reinvestigated following referral to a haematologist for advice on the management of pregnancy. The lupus anticoagulant was still undetectable, the anticardiolipin tests were normal but a test for the prothrombin 20210A allele showed that she was heterozygous for this abnormality.

\section{Discussion}

Three possible causes could, at least theoretically, be contributing to the development of the retinal vein occlusion in this woman: the previous lupus erythematosus, the oral contraceptive and the prothrombin 20210A allele. The episode of lupus erythematosus occurred 4 years earlier but since withdrawing the offending drug, tetracycline, there has been no clinical or laboratory marker of recurrence.

This patient was taking the combined oral contraceptive pill at the time of the retinal vein occlusion. We believe that, on current evidence, it is not possible to say whether the contraceptive pill in isolation is a risk factor for retinal vein occlusion.

The prothrombin 20210A allele was first reported to be associated with venous thrombosis in November 1996. It is due to a $G$ to $A$ transition at position 20210 of the prothrombin gene. Although this is in the 3 untranslated region of the gene, it is at the terminal position where the poly-A tail attaches prior to mRNA translation. This transition stabilises the mRNA molecule leading to increased prothrombin concentration and increased thrombotic risk. ${ }^{2}$ The prothrombin 20210A allele is found in $1 \%$ of the normal population and in $6 \%$ of patients with thrombosis. ${ }^{2,3}$

We can not be certain as to whether the prothrombin defect was a causative factor in this woman's retinal vein occlusion, but in combination with the oral contraceptive it could have been. Further studies are required to define the role of the contraceptive pill and inherited prothrombotic defects in the aetiology of retinal vein occlusion.

\section{References}

1. Kirwan JF, Tsaloumas MD, Vinall H, Prior P, Kritzinger EF, Dobson PM. Sex hormone preparations and retinal vein occlusion. Eye 1997;11:53-6.

2. Poort SW, Rosendaal FR, Reitsma PH, Bertina RM. A common genetic variation in the 3-untranslated region of the prothrombin gene is associated with elevated plasma prothrombin levels and an increase in venous thrombosis. Blood 1996;88:3698-703.

3. Makris M, Preston FE, Beauchamp NJ, et al. Co-inheritance of the 20210A allele of the prothrombin gene increases the risk of thrombosis in subjects with familial thrombophilia. Thromb Haemost 1997;78:1426-9.

Matthew Edwards

Simon Longstaff

Department of Ophthalmology

Royal Hallamshire Hospital

Glossop Road Sheffield S10. 2JF, UK

Mike Makris

Royal Hallamshire Hospital

Sheffield, UK 